\title{
Investigating the neuroanatomic wiring for emotions and homeostasis
}

\author{
Itopa Ajayi
}

$\mathrm{T}$ he expression of emotions involves modulating motor and autonomic functions. However, there is a gap in the understanding of the neuroanatomical link between emotions and homeostasis: forebrain and brainstem connectome.

The Pavlovian fear-conditioning paradigm has served as a useful model for investigating emotional behaviours. Using this paradigm, the amygdala, hypothalamus and periaqueductal gray (PAG) were identified as neuronal fields critical in mediating anxiety-related emotional processes. My research into the descending circuits that subserve the modulation of sighs - a significant autonomic indicator of emotional state - also demonstrated a role of the ventral hippocampus ( $\mathrm{VH}$ ) and the medial nucleus of the amygdala $(\mathrm{MeA})$ in mediating descending autonomic control. These pieces of evidence coupled with the known projections of the MeA to the PAG, which, in turn targets the nucleus retroambiguus of the rostral ventrolateral medulla, offer a patent pathway for limbic modulation of the autonomic nervous system. Through this tract, cardiorespiratory rhythm can be modulated during the expression of an emotional behaviour. However, the functional network that concurrently modulates emotions and homeostasis is only complete with the hypothalamus.

Research has shown that emotions such as rage can be generated on a precolliculi pre-hypothalamic decerebrate animal by stimulating specific neuron populations within the hypothalamus. Activating those nuclei also modulates the autonomic nervous system. These deductions suggest that the hypothalamus contains intrinsic circuits that are recruited in the functional network that orchestrates emotions while concurrently modulating sympathetic outflow. Thus, the hypothalamus is a crucial component in any debate evaluating the neuroanatomic wiring of emotions and homeostasis.

Recently, some Stanford researchers ${ }^{[1]}$ demonstrated the presence of overlapping glutamatergic nuclei responsible for regulating defensive behaviours. Their research involved exposing experimental subjects to homeostatic threats while monitoring changes to gross motor behaviours and hypothalamic gene expressions consistent with the manifestations of specific emotions. A significant finding from the study includes the demonstration of defensive reactions - too sudden to result from a hormonal feedback system following transient changes to homeostasis. A brain-wide two-photon $\mathrm{Ca}^{2+}$ imaging revealed the activation of discrete neuron populations in the hypothalamus, in addition to fore and hindbrain activations in the course of the emotions. Interestingly, the study also reported that fibres of the activated hypothalamic neurons converged on spinal projecting glutamatergic premotor neurons in the midbrain and hindbrain.

Juxtaposing these evidences suggests that the hypothalamus possesses the capacity to generate emotional responses of rage. Additionally, the hypothalamus mediates the top-down transmission of emotion and modulates the accompanying homeostatic-related signals. The hypothalamus is, therefore, a significant player in any discussion of emotions and homeostasis Furthermore, it must be noted that multiple nuclei function synchronously in the circuit physiology of a complex cascade of events to produce emotions, thoughts, memory and consciousness while modulating autonomic outflow. Anatomic experiments using retrograde and anterograde tracers, and indeed, recent $\mathrm{Ca}^{2+}$ and evolving non-invasive imaging techniques, offer tremendous insights into neuron connectome for emotions and homeostasis. However, to adequately investigate the neuroanatomic wiring for system-based functions, a cogent approach combining classical anatomic techniques with physiologic perturbations of inhibition, disinhibition and optogenetics, amongst other methods is required. It is only by creating a nexus between anatomic and physiologic techniques that we can objectively associate a neuronal circuit to specific motor behaviours and emotions.

\section{Reference}

[1] Lovett-Barron, M., Chen, R., Bradbury, S., Andalman, A. S., Wagle, M., Guo, S., Deisseroth, K. 2020. Multiple convergent hypothalamus-brainstem circuits drive defensive behavior. Nature Neuroscience, https://doi.org/10.1038/s41593-020-0655-1

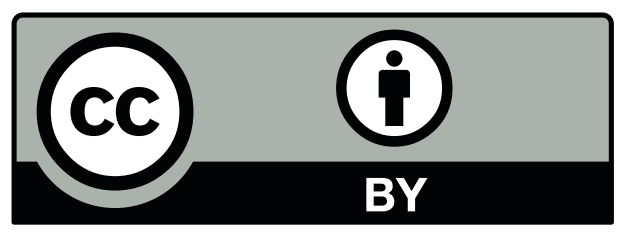

This article is an Open Access article distributed under the terms and conditions of the Creative Commons Attribution (CC BY) 4.0 International license (http://creativecommons.org/licenses/by/4.0/).

For correspondence

Itopa Ajayi, PhD

Department of Veterinary Anatomy

University of Abuja, Nigeria

Email: e.itopa@uniabuja.edu.ng 